\title{
Anomalous Dielectric Behavior of Water in Ionic Newton Black Films
}

\author{
Jordi Faraudo* and Fernando Bresme ${ }^{\dagger}$ \\ Department of Chemistry, Imperial College London, Exhibition Road, London, SW7 2AZ, United Kingdom
}

(Received 15 December 2003; published 10 June 2004)

The electrostatics of two charged surfactant layers in aqueous media (surfactant/water/surfactant films) is investigated using molecular dynamics simulations. In the films studied (with a surfactantsurfactant distance from $\sim 35 \AA$ to contact) we observe an anomalous dielectric response of water. The electrostatic potential $\phi(z)$ inside the aqueous core of the films (containing bulk water with $\rho=$ $1 \mathrm{~g} / \mathrm{cm}^{3}$ ) is completely different from that expected for a film containing a dielectric medium with the dielectric constant of water. In addition, our results are not consistent with a local relation between the water polarization $P_{z}(z)$ and the electric field $E_{z}(z)$. The polarization $P_{z}(z)$ is maximum at the interfaces (due to solvent molecules forming part of the structure of the surfactant layers) and decays from the interfaces inside the aqueous core with a decay length of order of $\sim 10 \AA$.

Electrostatic interactions in aqueous media between surfaces or interfaces possessing net electric charge play an essential role in many biological and industrial processes. Examples of such situations are water-air interfaces with adsorbed ionic surfactants, colloids, and biological membranes. The classical description of these electrostatic interactions is based on the PoissonBoltzmann equation (PB) [1-3]. The PB equation is accurate in many situations but has serious limitations at small distances between the charged surfaces or interfaces. At these distances, some effects not included in the PB equation may be relevant. Some of these effects are the finite size of the ions, the discrete nature of the charges of the interface, or the correlations between ions (see, for example, Chap. 12 in [1]). Many theoretical and simulation studies have been focused on these effects, and the literature on the subject is immense (many references can be found in [1-4]).

Another important hypothesis assumed in the $\mathrm{PB}$ equation is that the solvent (water) is characterized by a dielectric constant $\varepsilon_{r}$. Many studies have been devoted to the analysis of this hypothesis (see [5] for a review and references), some of them claiming that water in these systems has a more complex dielectric response. For example, Marcelja and coworkers [6] have developed a theory in which water confined between charged interfaces has a nonlocal dielectric response: the relation between the electric field $\vec{E}$ and the polarization $\vec{P}$ is determined by the condition of minimum electrostatic free energy and a local dielectric constant simply does not exist. In this theory, the structure acquired by water near charged surfaces or interfaces completely alters its dielectric response. However, these visions about the dielectric response of water are not supported by the conclusions of the critical review of theoretical, experimental, and computational work performed by Israelachvili and Wennerström [5]. These authors claim that the dielectric constant and the diffusivity of water near charged soft surfaces are the same as in bulk water, and only the first layer of water molecules (in contact with the charged interfaces) has different properties than bulk water. In their theory of the role of hydration in biological and colloidal interactions [5], entropic considerations rather than water structure play a key role.

In order to test these proposals, Marcelja [7] has computed the interaction force between two equally charged rigid planar walls in aqueous electrolyte solution. At short separations $(\sim 20 \AA)$, significant corrections to the PB predictions appear due to the hydration of the ions $[7,8]$. It is important to remark that these results apply to the case of interaction between impenetrable rigid charged walls, but the interactions between soft surfaces and interfaces with net electric charge are expected to be very different $[5,9]$. In this important case, relevant information has been obtained from experiments in Newton black films (NBF) $[9,10]$. NBFs are very thin films of size $\sim 40 \AA$ consisting of an aqueous core $(\sim 7.5 \AA)$ with water and ions sandwiched between two layers of adsorbed surfactant [10]. There are important differences between the surfactant layers of a NBF and the planar impenetrable charged walls usually considered in the mentioned theoretical models [5,9]: surfactant layers are rough; they experience undulations and protrusions, which may have an effect over charge distribution; water molecules can penetrate inside the layers, even becoming part of the layer as hydration water; etc. It is also important to recall that the structure of the solvent confined between two rigid walls is very different than the structure of water inside a NBF.

Our objective in this Letter is precisely to test the basic theoretical concepts in the theories on the role of water structure on electrostatic interactions mentioned in the previous paragraphs by performing computer simulations of a realistic model of a NBF. This analysis is made using molecular dynamics simulations, which need to be performed by taking advantage of supercomputing facilities. 
In this work, we consider water films of different sizes sandwiched between two layers of adsorbed sodium dodecyl sulfate (SDS) surfactant $\left[\mathrm{Na}^{+} \mathrm{CH}_{3}\left(\mathrm{CH}_{2}\right)_{11} \mathrm{OSO}_{3}^{-}\right]$. We have chosen this widely used detergent because NBFs consisting of SDS/water/SDS have been investigated experimentally in detail [10]. Water is modeled using the SPC/E model (an extended simple point charge water, a classical well-known model of water) [11], and the model and force field employed for the surfactant are described in detail in [12]. The simulation results obtained using these models for water and SDS reproduce with good accuracy the available structural experimental data on NBF [12]. In our simulations, we consider two layers containing $N_{\mathrm{SDS}} / 2 \mathrm{DS}^{-}$cations, each one $\left(N_{\mathrm{SDS}}=128\right)$ with a surface area per surfactant of $A / N_{\mathrm{SDS}}=33 \AA^{2}$ (corresponding to the value found in experiments [10]) separated by water containing $N_{\mathrm{SDS}} \mathrm{Na}^{+}$ions (which can be adsorbed at the interface). In order to change the separation $H$ between both surfactant layers, we consider simulations with a different number of water molecules $N_{w}$. Extensive technical details about our simulations can be found in Ref. [12], and some pictures and a movie of our simulations can be found in the electronic auxiliary material (EPAPS) [13]. For simplicity, here we will analyze only the results for some representative film sizes. In Fig. 1(a) we show the results obtained for the free charge distribution $\rho_{f}(z)$ due to the $\mathrm{DS}^{-}$and $\mathrm{Na}^{+}$ions averaged over $1.5 \mathrm{~ns}$ for a system with $N_{w} / N_{\mathrm{SDS}}=11.94$. In the same figure, we also show the mass density of water in the film. The system has a core of water with density $\rho \approx$ $1 \mathrm{~g} / \mathrm{cm}^{3}$ sandwiched between two interfaces with adsorbed $\mathrm{DS}^{-}$and $\mathrm{Na}^{+}$ions. This aqueous core contains approximately $\sim 10 \%$ of the $\mathrm{Na}^{+}$ions. In the two interfacial regions (of size $\sim 8 \AA$ ), $\rho_{f}(z)$ has a complex structure, reflecting the distributions of the adsorbed $\mathrm{DS}^{-}$ cations and the $\mathrm{Na}^{+}$ions accumulated near the charged interface. It is clear from Fig. 1(a) that the adsorbed surfactant layers contain solvation water. We have computed the mean electric field $E_{z}$, the dielectric displacement $D_{z}(z)$, and the electrostatic potential $\phi(z)$ (averaged over the $X Y$ plane) using the Gauss theorem:

$$
\begin{gathered}
E_{z}(z)=-\frac{d \phi}{d z}=\frac{1}{\varepsilon_{0}} \int_{-\infty}^{z} \rho\left(z^{\prime}\right) d z^{\prime}, \\
D_{z}(z)=\int_{-\infty}^{z} \rho_{f}\left(z^{\prime}\right) d z
\end{gathered}
$$

where $\rho(z)$ is the charge distribution due to all the charges present in the system. In Fig. 1(b) we show the electrostatic potential $\phi(z)$ for this system. The potential has strong oscillations at the interfaces, but it is nearly constant in the core (indicating a zero field in this region). Also, note that $\phi(z)$ is slightly asymmetric with respect to $z \rightarrow-z$ due to the asymmetries present in $\rho_{f}(z)$ [see Fig. 1(a)]; therefore spontaneously formed charge asymmetries between both layers can survive up to time scales of the order of the nanosecond.
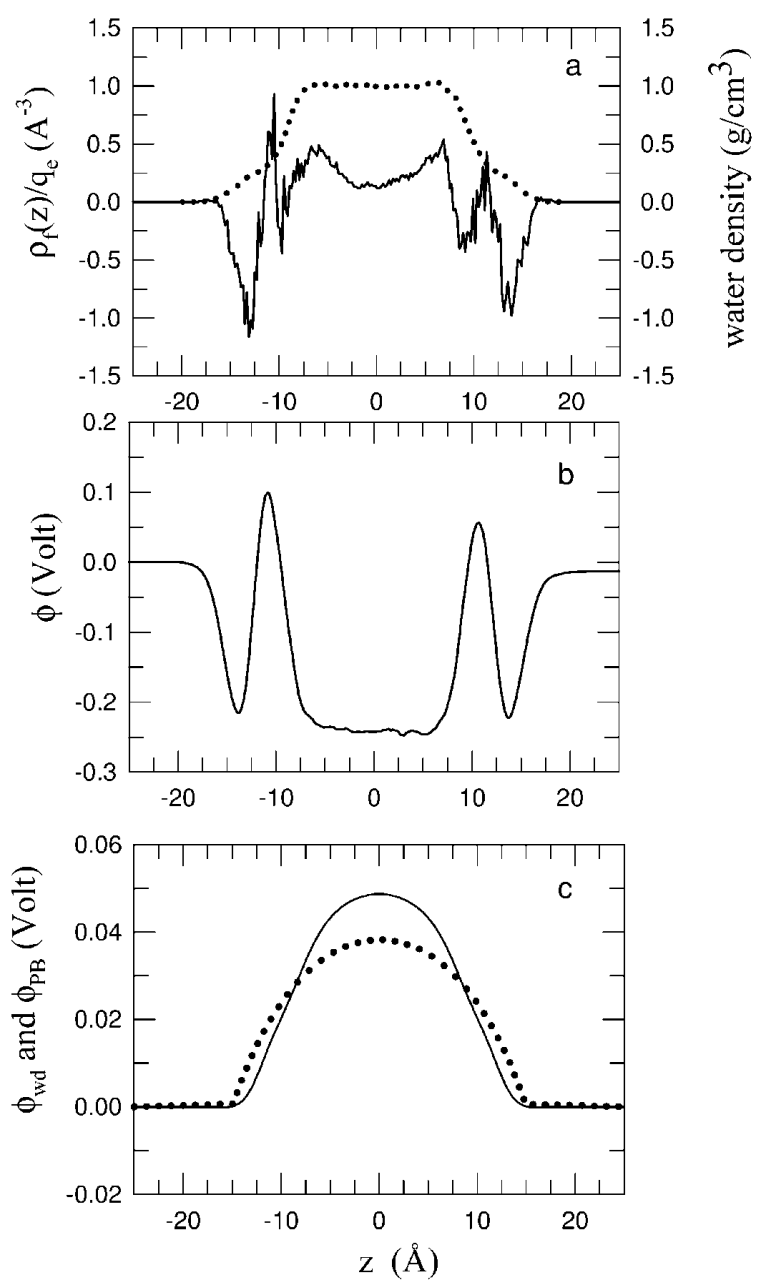

FIG. 1. Simulation results for a system with $N_{w} / N_{\mathrm{SDS}}=$ 11.94. (a) Solid line: charge distribution $\rho_{f}(z) / q_{e}$ due to the $\mathrm{DS}^{-}$and $\mathrm{Na}^{+}$( $q_{e}$ is the electronic charge); dots: density of water in $\mathrm{g} / \mathrm{cm}^{3}$. (b) Electrostatic potential $\phi(z)$ computed using Eq. (1). (c) Solid line: $\phi_{w d}$ computed assuming that water behaves as an ordinary dielectric [Eq. (3)]; dotted line: solution of the PB equation.

In order to determine the role of the solvent in the observed $\phi(z)$, let us compute a new electrostatic potential $\phi_{w d}$ defined by

$$
-\frac{d \phi_{w d}}{d z} \equiv \frac{1}{\varepsilon_{0} \varepsilon_{r}} D_{z}(z)=\frac{1}{\varepsilon_{0} \varepsilon_{r}} \int_{-\infty}^{z} \rho_{f}\left(z^{\prime}\right) d z,
$$

where $\varepsilon_{r}$ is the dielectric constant of ordinary bulk water (for the SPC/E model of water at $298 \mathrm{~K}$, one has $\varepsilon_{r} \simeq 70$ [14]). The quantity $\phi_{w d}$ is simply the electrostatic potential generated by the distribution of charge $\rho_{f}(z)$ due to ions and surfactants (obtained in the simulations) immersed in a dielectric medium with dielectric constant $\varepsilon_{r}$. In the calculation of $\phi_{w d}$, we replace the actual solvent distribution obtained in simulations by a dielectric medium with relative dielectric constant $\varepsilon_{r}$. In other words, the quantities $\phi(z)$ and $\phi_{w d}(z)$ are equal if the solvent in the simulations has the same dielectric response of 
ordinary SPC/E bulk water. The result for the symmetrized $\phi_{w d}(z)$ is shown in Fig. 1(c). A comparison of Figs. 1(b) and 1(c) by direct inspection shows striking differences between $\phi(z)$ and $\phi_{w d}(z)$. It is very important to recall that these differences are evident even in the central region that contains a slab of water with density $\rho \approx 1 \mathrm{~g} / \mathrm{cm}^{3}$, expected to behave as bulk water. In this region, the actual potential $\phi(z)$ fluctuates slightly around a constant value (so $E_{z} \approx 0$ ), whereas the potential $\phi_{w d}(z)$ is far from being constant, indicating an electric field pointing towards the nearest surfactant layer [see Fig. 1(c)] and has a maximum at $z \simeq 0$. These impressive differences clearly show that the dielectric response of water inside the film is very different from that expected for a dielectric medium characterized by $\varepsilon_{r}$. In Fig. 1(c) we also show the prediction $\phi_{\mathrm{PB}}(z)$ of the $\mathrm{PB}$ equation, assuming two uniformly charged planar walls separated a distance $H \simeq 28 \AA$ [1]. It is remarkable that the PB equation predicts a result not essentially different from the quantity $\phi_{w d}(z)$. Therefore, the differences between the predictions of the $\mathrm{PB}$ equation $\left[\phi_{\mathrm{PB}}(z)\right]$ and the simulation results $(\phi(z))$ are mainly due to the fact that water inside the film has an anomalous dielectric response. Other effects such as correlations between charges, finite ion sizes, or discrete nature of charges in the adsorbed layers seem to have only secondary importance in the electrostatics of this specific system. Also, note that, in the simulated system roughness, undulations and protrusions of the interface are expected to affect the charge distribution $\rho_{f}$, which has a complex structure [recall Fig. 1(a)]. However, the potential $\phi_{\mathrm{PB}}(z)$ is computed replacing the interfaces by charged planes. The comparison between $\phi_{w d}(z)$ and $\phi_{\mathrm{PB}}(z)$ [see Fig. 1(c)] suggests that the electrostatic potential is not strongly influenced by this complex structure of the charged interface.

The dielectric response of the medium (the contribution to the observed electric field due to water molecules) is given by the polarization $P_{z}(z)$ defined as

$$
P_{z} \equiv D_{z}-\varepsilon_{0} E_{z}=\int_{-\infty}^{z}\left[\rho\left(z^{\prime}\right)-\rho_{f}\left(z^{\prime}\right)\right] d z^{\prime} .
$$

In Fig. 2 we show the fields $E_{z}$ and $P_{z}$ for three different systems with $N_{w} / N_{\mathrm{SDS}}=11.9, \quad N_{w} / N_{\mathrm{SDS}}=22$, and $N_{w} / N_{\text {SDS }}=4.36$, respectively. The electric field $E_{z}$ oscillates at the interfaces with a maximum value around $\left|E_{z}\right| \approx 2 \times 10^{9} \mathrm{~V} / \mathrm{m}$ and vanishes inside the aqueous core of the films [see Figs. 2(a) and 2(b)], so the electric field is confined at the interfaces. The polarization of water at the interfaces is very strong in all the systems, of order $\left|P_{z}\right| / \varepsilon_{0} \approx 5 \times 10^{9} \mathrm{~V} / \mathrm{m}$ [see Figs. 2(a) $\left.-2(\mathrm{c})\right]$. This value for the polarization implies a very high degree of ordering of water molecules at the interface. This can be seen by noting that the dipolar moment of a SPC/E water molecule is $\mu=7.8 \times 10^{-30} \mathrm{C} \mathrm{m}$; so the maximum polarization that can be expected at an interface of size $h \approx$ $8 \AA$ containing $N_{s w}$ water molecules is $P_{z} / \varepsilon_{0} \approx$ $N_{s w} \mu /\left(A h \varepsilon_{0}\right) \approx 10^{9} \mathrm{~V} / \mathrm{m}$. This polarization acquired at 236102-3
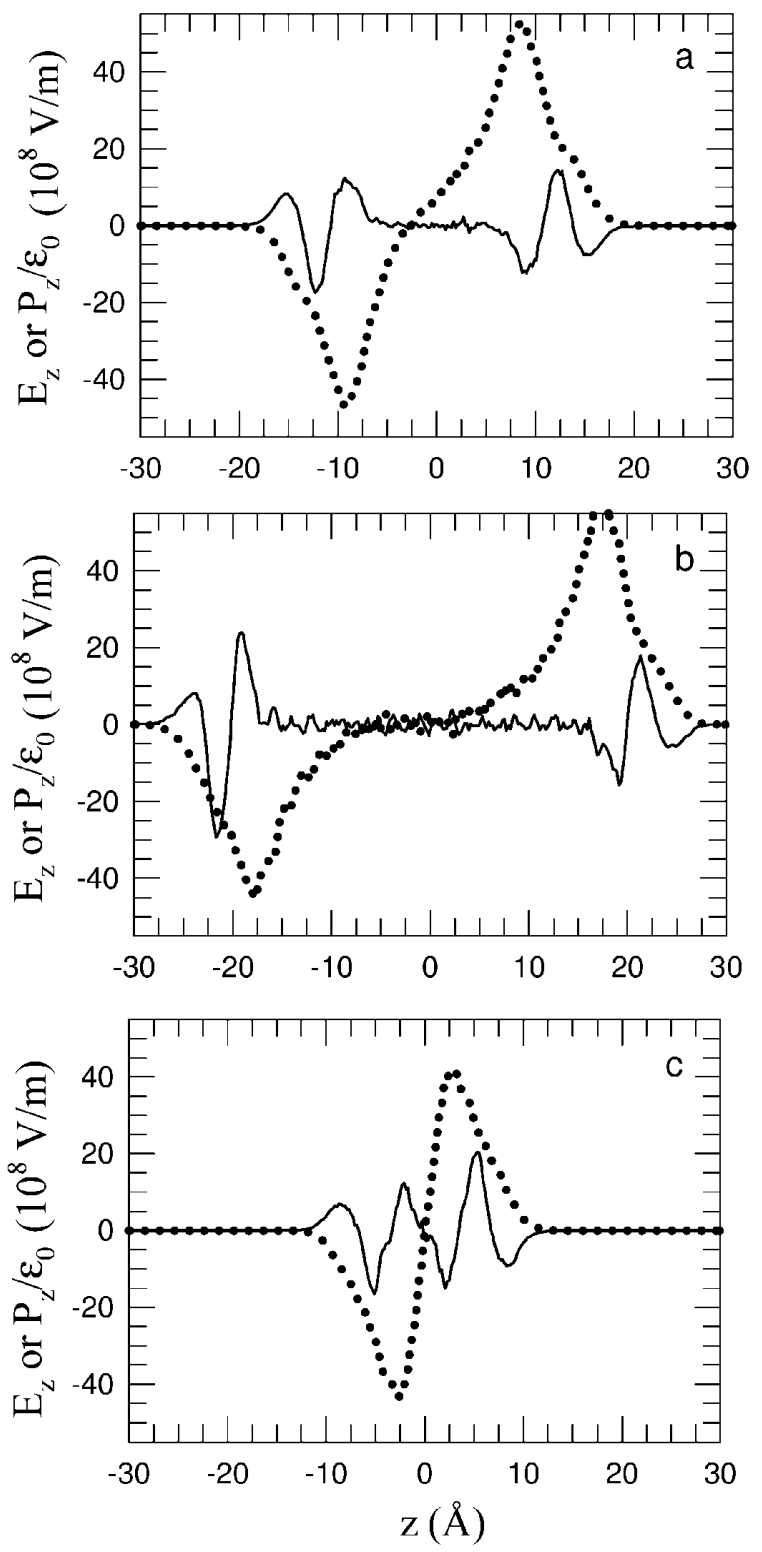

FIG. 2. Fields $E_{z}$ (solid line) and $P_{z} / \epsilon_{0}$ (dotted line) in $10^{8} \mathrm{~V} / \mathrm{m}$. (a) Simulation with $N_{w} / N_{\mathrm{SDS}}=11.94$, (b) $N_{w} / N_{\text {SDS }}=22$, and (c) $N_{w} / N_{\text {SDS }}=4.37$.

the interfaces is due to the solvation water forming part of the structure of the surfactant layers. This can be shown by noting that all the systems have the same polarization at the interface as the film with the interfaces at contact [water content of $N_{w} / N_{\mathrm{SDS}}=4.36$; see Fig. 2(c)], which does not contain any bulk water but contains only hydration water. It is worth noting that the structural details of this film with $N_{w} / N_{\mathrm{SDS}}=4.36$ [12] are in good agreement with the films spontaneously formed in ambient conditions in the experiments of Ref. [10].

In Figs. 2(a) and 2(b) we can observe how this high polarization $P_{z}$ acquired at the interfaces is propagated up to the bulk water molecules, decaying in the central region of the film in a typical distance of $\sim 10 \AA$. This observed polarization of water at the aqueous core 
suppresses completely the field created by the charge density $\rho_{f}(z)$ due to the $\mathrm{DS}^{-}$and $\mathrm{Na}^{+}$ions (see Fig. 1), so the electric field vanishes in the aqueous core because of the polarization of water. This result of a decaying polarization from the interface is completely different from the polarization expected in an ordinary dielectric, in which the local relation $P_{z}=\left(\varepsilon_{r}-1\right) \varepsilon_{0} E_{z}$ holds. In our simulation results (see Fig. 2), no local relation between the fields $P_{z}$ and $E_{z}$ seems to exist [for example, note that in the central region of Fig. 2(a), between $z \approx$ $-7.5 \AA$ and $z \approx 7.5 \AA, E_{z} \approx 0$, whereas $P_{z}$ changes smoothly from $P_{z} / \varepsilon_{0} \approx-5 \times 10^{9} \mathrm{~V} / \mathrm{m}$ to $P_{z} / \varepsilon_{0} \approx 5 \times$ $\left.10^{9} \mathrm{~V} / \mathrm{m}\right]$.

After analyzing the obtained electrostatic potential and fields, the remaining question is why water molecules arrange themselves in order to give this unexpected dielectric response instead of behaving according to the ordinary dielectric response of bulk water. The answer to this question is probably complex, but a simple insight can be obtained by computing the electrostatic free energy difference between both dielectric behaviors of water. Let us define the following quantity:

$$
\Delta F \equiv \frac{A}{2} \int_{-\infty}^{\infty} P_{z} E_{z} d z-\frac{A}{2} \frac{\varepsilon_{r}-1}{\varepsilon_{r}^{2} \varepsilon_{0}} \int_{-\infty}^{\infty} D_{z}^{2} d z .
$$

In Eq. (5), the first integral is the work needed to insert the water distribution observed in the simulation into a system originally without water but containing the charge distribution $\rho_{f}(z)$, maintaining a fixed charge distribution $\rho_{f}(z)$ and temperature $T$. The second integral is the work needed to insert a dielectric characterized by $\varepsilon_{r}$ [hence with polarization $\left(\varepsilon_{r}-1\right) D_{z} / \varepsilon_{r}$ ] in a system with the charge distribution $\rho_{f}(z)$, maintaining a fixed charge distribution $\rho_{f}(z)$ and temperature $T$. Hence, the quantity $\Delta F$ quantifies how favorable the observed dielectric response of water is in the films as compared with a solvent characterized by a dielectric constant $\varepsilon_{r}$. We obtain $\Delta F=-0.28 N_{w} k_{B} T$ for $N_{w} / N_{\text {SDS }}=22, \Delta F=$ $-0.29 N_{w} k_{B} T$ for $N_{w} / N_{\mathrm{SDS}}=11.94$, and $\Delta F=$ $-0.26 N_{w} k_{B} T$ for $N_{w} / N_{\mathrm{SDS}}=4.36$. The results for $\Delta F$ are negative but small. Water in this system has a thermodynamic preference for the observed dielectric response rather than to behave as an usual dielectric medium.

In conclusion, our computer simulations of two charged interfaces in aqueous media (ionic surfactant/ water/ionic surfactant films with a distance between interfaces from $\sim 35 \AA$ to contact) show an anomalous dielectric response of water. Our present results show that the region influenced by the charged surfactant layers extends up to a distance of order $\sim 10 \AA$. Solvent molecules acquire a high polarization at the charged surfactant layers, which decays from the interfaces with a decay length of order of $\sim 10 \AA$. This observed polarization is not consistent with a local relation between the polarization of the solvent and the electric field. As a result of this decaying polarization, the electrostatic potential $\phi(z)$ inside a film containing a small amount of bulk water ( $\sim 20 \AA$ ) is completely different from that expected if water behaves as an usual dielectric. We also show that this effect is a significant source of deviation between the predictions of the classical Poisson-Boltzmann equation and the observed electrostatic potential.

We hope that these new findings finally clarify the question debated in the literature (see, for example, [5] for a review) about the dielectric behavior of water in these complex systems containing two charged soft interfaces. We expect that our findings will stimulate new theoretical research on the important subject of electrostatic forces between charged interfaces in aqueous media.

This work has been supported by EPSRC Research Grant No. GR/R39726/01. Simulations were performed at the HPCx Supercomputing Center (Edinburgh, U.K.) under the Materials Chemistry Consortium (U.K.). One of the authors (J. F.) also acknowledge financial support from the Spanish Government under Grant No. BFM20000351-C03-01.

*Present address: Departament de Fisica, Universitat Autonoma de Barcelona, Facultat de Ciencies, E-08193 Bellaterra, Spain.

Electronic address: Jordi.Faraudo@uab.es

†Electronic address: f.bresme@imperial.ac.uk

[1] J. Israelachvili, Intermolecular and Surface Forces (Academic Press, London, 1991), 2nd ed.

[2] D. F. Evans and H. Wennerström, The Colloidal Domain (Wiley-VCH, New York, 1999), 2nd ed.

[3] H.T. Davis, Statistical Mechanics of Phases, Interfaces and Thin Films (VCH, New York, 1996).

[4] B.W. Ninham, Adv. Colloid Interface Sci. 83, 1 (1999).

[5] J. Israelachvili and H. Wennerstrom, Nature (London) 379, 219 (1996).

[6] S. Marcelja and N. Radic, Chem. Phys. Lett. 42, 129 (1976); D.W. R. Gruen and S. Marcelja, J. Chem. Soc. Faraday Trans. 2 79, 225 (1983).

[7] S. Marcelja, Nature (London) 385, 689 (1997).

[8] J. Israelachvili and H. Wennerstrom, Nature (London) 385, 690 (1997).

[9] V. Bergeron, J. Phys. Condens. Matter 11, 215 (1999).

[10] O. Bélorgey and J. J. Benatar, Phys. Rev. Lett. 66, 313 (1991).

[11] H. J. C. Berendsen, J. R. Grigera, and T. P. Straatsma, J. Phys. Chem. 91, 6269 (1987).

[12] F. Bresme and J. Faraudo, Langmuir 20, 5127 (2004).

[13] See EPAPS Document No. E-PRLTAO-92-041422 for pictures and movies of simulations of a system. A direct link to this document may be found in the online article's HTML reference section. The document may also be reached via the EPAPS homepage (http://www.aip.org/ pubservs/epaps.html) or from ftp.aip.org in the directory /epaps/. See the EPAPS homepage for more information.

[14] P. Höchtl et al., J. Chem. Phys. 109, 4927 (1998). 